\title{
System identification of tumor growth described by a mixed effects model
}

\author{
T. Bastogne ${ }^{*}$ A. Samson ${ }^{* *}$ S. Mézières-Wantz ${ }^{* * *}$ \\ P. Vallois ${ }^{* * *}$ S. Pinel ${ }^{* * *}$ M. Barberi-Heyob ${ }^{\dagger}$ \\ * Centre de Recherche en Automatique de Nancy (CRAN), \\ Nancy-Université, CNRS, BP 239, F-54506 Vandouvre-lès-Nancy \\ Cedex, France, (thierry.bastogne@cran.uhp-nancy.fr). \\ ** Laboratoire MAP5, CNRS, Université Paris Descartes, Paris \\ *** Institut de Mathématiques Elie Cartan, Nancy-Université, CNRS, \\ BP 239, F-54506 Vandouvre-lès-Nancy Cedex, France \\ **** EA 4001, Nancy-Université \\ $\dagger$ Centre de Recherche en Automatique de Nancy (CRAN), \\ Nancy-Université, CNRS, Centre Alexis Vautrin, 54511 \\ Vandouvre-lès-Nancy Cedex, France
}

\begin{abstract}
System identification of treated tumor growth is addressed in this paper. Three main difficulties are examined: (i) the determination of a suited dynamical model structure (modeling problem), (ii) the inter-individual variability of the therapeutic responses (population identification problem or longitudinal data analysis) and (iii) the effects of some categorical factors on the model parameters. To solve these problems, a mixed effect model of tumor growth, a two step identification approach and an estimation algorithm based on expectation maximization, are proposed and applied to in vivo data. A double effect of treatments on the tumor volume responses is pointed out. *
\end{abstract}

* 15th Symposium on System Identification, SYSID’09, Saint-Malo, France, July 6-8 2009

Keywords: System identification; Biomedical systems; Statistical inference; Variability; Uncertain dynamic systems

\section{INTRODUCTION}

New cancer therapies cannot be developed efficiently unless it is possible to predict the therapeutic effects on the tumor growth, in a reliable, robust and reproducible manner. Reliability, robustness and reproducibility of the therapeutic outcomes can be expressed as control problems. In de Pillis and Radunskaya (2001); Tervo et al. (2007); Dua et al. (2008), control theory was applied to cancer therapies. These control strategies require to consider mathematical modeling of tumor growth. Such modeling studies were developed in Fischer (1971); Thompson and Brown (1987); Adam and Bellomo (1997); Drasdo and Höhme (2005). The growth models proposed were most of time kinetic and not dynamic. The effect of input variables on the growth response, like the damage caused by the treatment, was not explicitly taken into account. In this control framework, the mathematical model of the system must be simple enough so that it can be analyzed with available mathematical techniques, and accurate enough to describe the important aspects of the relevant dynamical behavior. Moreover, size of the tumor growth data sets is generally small which reasonably limits the model complexity. The first problem addressed herein is thus to determine a dynamic model structure able to correctly fit dynamics of tumors and simple enough to be used in a control perspective.
The second main issue is the inter-individual variability of treatment responses. Indeed, in vitro as well as in vivo experiments are always repeated to assess the reproductiveness degree of the experimental responses. Quantifying the inter-individual variability of the model parameters is a crucial point, in particular in the analysis of the biological model. The problem is not to identify one biological system but a batch of similar biological systems. The associated inference problem is based on longitudinal data, i.e. data observed over subjects as well as time. A first modeling approach consists in estimating as many models as the number of subjects, and then to statistically compute the empirical distribution of the model parameters. The main drawback of this approach is to increase the parameterto-observation ratio. Another approach is to use mixed effects models. The latter have been largely employed for the identification of static systems but their use in dynamic system identification problems is much more recent (Lindstrom and Bates (1990); Samson et al. (2006, 2007)).

A third typical problem in experimental biology is to assess the effects of biological factors on the model parameters. In other terms, that consists to identify some deterministic causes of the response variability and their influence degree. Some of these factors can be continuous or categorical. In this application, the treatment mode (radiotherapy and combined chemotherapy-radiotherpy) is considered as a categorical factor. 
In this paper, the system identification of treated tumor growth described by a mixed model from in vivo longitudinal data sets is proposed. The analysis of the mixed model is performed using the Monolix ${ }^{1}$ software. A basic model structure of tumor growth based on cell population dynamics, completed by a treatment function, is proposed in section 2. The mixed effect model is presented in section 3. The experimental setup and estimation data sets are described in section 4 . The SAEM algorithm used for the identification of mixed effects models is presented in section 5. Identification results are then shown and a discussion is held in section 6 . Conclusions and perspectives of this work are drawn in section 7 .

\section{DETERMINISTIC TUMOR GROWTH MODELING}

Table 1. Main notations

\begin{tabular}{ll}
\hline Symb. & Description \\
\hline$t$ & time \\
$v(t)$ & model output (explained volume) \\
$y(t)$ & measured volume of the tumor \\
$x(t)$ & number of cancer cells of the tumor \\
$x_{n}(t)$ & number of cells born during $[0 ; t]$ \\
$x_{m}(t)$ & number of dead cells during $[0 ; t]$ \\
$u(t)$ & undergone damage induced by the treatment \\
$\delta(t)$ & Dirac delta function (impulse) \\
\hline$x_{0}$ & initial number of cells (in the graft) \\
$d$ & number of parameters \\
$\Theta$ & vector of the model parameters in $\mathbb{R}^{d}$ \\
$n$ & number of observation \\
$r$ & number of repeated experiments \\
\hline
\end{tabular}

\subsection{Non treated tumor: an autonomous system}

A tumor can be regarded as a population of cancer cells. Its growth may then be described by a model of cell population dynamics defined by

$$
\begin{aligned}
x(t) & =x_{n}(t)-x_{m}(t) \\
\dot{x}_{n}(t) & =f_{n}(x(t)) \\
\dot{x}_{m}(t) & =f_{m}(x(t), u(t)) \\
x_{n}(0) & =x_{0} \text { and } x_{m}(0)=0 .
\end{aligned}
$$

$f_{n}(\cdot), f_{m}(\cdot)$ are two mathematical functions associated with usual growth models presented in Walter and Lamprecht (1978) and Thompson and Brown (1987). One of the most commonly used growth model is probably the Malthusian model, sometimes called the simple exponential growth model, in which reproduction and mortality are supposed to be proportional to the total number of cells $(x)$ in the tumor. In this case, reproduction and mortality dynamics are defined by

$$
\begin{aligned}
\dot{x}_{n}(t) & =a x(t) \\
\dot{x}_{m}(t) & =b x(t) .
\end{aligned}
$$

The model output variable $v$ is the tumor volume and is defined as proportional to the number of cancer cells in the tumor,

$$
v(t)=\gamma x(t)
$$

\footnotetext{
1 Monolix (MOdèles NOn LInéaires à effets miXtes) is a software dedicated to the analysis of nonlinear mixed effects models. It is designed for academic and industrial researchers or engineers, concerned with the analysis of longitudinal data in various fields. Monolix is developed by the Monolix group and is implemented in Matlab`. http://www.monolix.org
}

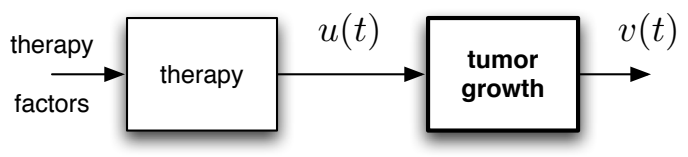

Fig. 1. Two consecutive phenomena: therapy and tumor growth

Solution of these differential equations is given by

$$
v(t)=v_{0} e^{\alpha t}=f_{0}(t) \quad \forall t \geq 0
$$

where $v_{0}=\gamma x(0)$ is the initial volume of the tumor. This equation describes the autonomous or natural mode of a (non treated) tumor growth. The parameter $\alpha=a-b$ is related to the mean frequency of the cancer cell cycle, and is defined positive. In other terms, a tumor is an unstable system. The vector of unknown parameters for this model is $\Theta=\left(v_{0}, \alpha\right)$.

\subsection{Treated tumor: a controlled system}

The anti-cancer treatment effect on the tumor volume is described by a dynamical function added to (8) to give

$$
v(s)=\frac{v_{0}}{(s-\alpha)}-\frac{\nu}{s(s+\nu)} e^{-\tau s} u(s),
$$

where $s$ is the Laplace variable. The first part of this equation describes the natural behavior of the tumor (equation (8)) while the second one corresponds to the forced mode (treatment effect). $\nu$ is a positive parameter related to the damage propagation velocity associated with the anti-cancer treatment. $\tau$ denotes a time delay between the end of the treatment and the beginning of its effects on the tumor size. $u(s)$ represents the undergone damage variable. In practice, $u$ is not exactly an input variable since it cannot be directly controlled. Indeed as shown in Figure 1, $u$ is the consequence (i.e. the output variable) of another process (radiation process for the radiotherapy, chemical process for the chemotherapy and photoreaction process for the photodynamic therapy) which is controlled by treatment factors (essentially doses). This preliminary process is not considered in this study since our objective is to model the general behavior of a tumor after any kind of treatment. Moreover, the damage variable $u$ cannot be measured online, i.e. without stopping the tumor growth (mouse sacrifice), which makes the tumor growth modeling from experimental data a blind system identification problem. However, one realistic assumption about $u$ can be stated. In practice, damage induced by the treatment are caused during a short period of time (typically $<1 \mathrm{~h}$ ) compared to the response time of the tumor growth dynamics (about several ten-day periods). As a consequence, $u(t)$ may be approached by a Dirac pulse, $u(t)=u_{0} \delta(t)$ where $u_{0}$ denotes the magnitude of tumor damage caused by the treatment. After application of the inverse Laplace transform to (9) with $u(s)=u_{0}$, we get

$$
v(t)=f_{0}(t)-f_{u}(t),
$$

with:

$$
\begin{aligned}
& f_{0}(t)=v_{0} e^{\alpha t} H(t) \\
& f_{u}(t)=u_{0}\left(1-e^{-\nu(t-\tau)}\right) H(t-\tau) .
\end{aligned}
$$

$H(t)=\int_{-\infty}^{t} \delta(z) d z$ is the Heaviside step function. Hereafter, the value of the explained volume $v_{i}\left(t_{j}\right)$ of the 
subject (mouse tumor) $i$ observed at time $t_{j}$ will be noted $v_{i j}$ to simplify reading. $\boldsymbol{\Theta}=\left(\begin{array}{lllll}v_{0} & u_{0} & \alpha & \nu & \tau\end{array}\right)$ is the vector of unknown model parameters.

\section{MIXED EFFECTS MODEL}

In practice, i.e. in in vivo context, uncertainty about the tumour growth is large and comes from several sources. Main causes are probably measurement errors, modeling approximations and biological variability, i.e. interindividual and uncontrolled variations of the response. Indeed, one particularity in experimental biology is the repetition of experiments on similar subjects in order to assess reproducibility of results. In this context, intersubject variations of responses are unavoidable and system identification becomes an inference problem from longitudinal data. The inference problem is not based on time series only but on longitudinal data (observed over time as well as over subjects). This particular issue can be solved by using mixed effects models. In our case, the mixed model of the tumor growth is given by:

$$
y_{i j}=v_{i j}\left(\boldsymbol{\Theta}_{i}\right)+a e_{i j} \quad \text { with } \quad e_{i j} \sim_{i . i . d .} \mathcal{N}(0,1)
$$

where $y_{i j} \in \mathbb{R}$ is the $j^{\text {th }}$ observation (measured volume) of subject $i, 1 \leq i \leq r$ with $r$ the number of subjects (repeated in vivo experiments), and $1 \leq j \leq n_{i}$ with $n_{i}$ the number of observations (time points) of subject $i$. As previously presented, $v_{i j}$ is the $j^{\text {th }}$ model output value (defined in (10)) of subject $i . e_{i j}$ is the within-group error. To deal with the inter-individual variability, the vector $\boldsymbol{\Theta}_{i}$ of individual random parameters is decomposed in fixed and random effects:

$$
\boldsymbol{\Theta}_{i}=\boldsymbol{\lambda}+\beta c_{i}+\boldsymbol{\eta}_{i} \text { with } \ln \left(\boldsymbol{\eta}_{i}\right) \sim_{i . i . d .} \mathcal{N}(0, \Omega)
$$

$\boldsymbol{\lambda}$ is an unknown vector of length $d$, composed of the nominal or mean values of the model parameters. $\beta$ is an unknown vector of coefficients. $c_{i}$ is a categorical covariate associated with the treatment mode, that takes the value 1 for radiotherapy and 2 for a combined chemotherapyradiotherapy. Fixed effects are defined in $\mu=(\boldsymbol{\lambda}, \beta) . \boldsymbol{\eta}_{i}$ denotes a vector of random effects. It is supposed that $e_{i j}$ and $\boldsymbol{\eta}_{i j}$ are mutually independent. $\Omega$ is the covariance matrix of the random effects and is defined herein as a diagonal matrix. Accordingly, the $l^{\text {th }}$ individual parameter $\theta_{i l}$ of subject $i$ can be decomposed as

$$
\theta_{i l}=\lambda_{l}+\beta_{l} c_{i}+\eta_{i l} \quad \text { with } \ln \left(\eta_{i l}\right) \sim_{i . i . d .} \mathcal{N}\left(0, \omega_{l}^{2}\right)
$$

where $\omega_{l}^{2}=\Omega(l, l)$. Finally, the model hyper-parameters to estimate from experimental data are gathered in $\boldsymbol{\psi}=$ $(\mu, \Omega, a)$.

\section{ESTIMATION METHOD BY THE SAEM ALGORITHM}

The parameter estimation of nonlinear mixed model is complex: the likelihood has no closed form because of the nonlinearity of the regression function in the individual parameters. Let us denote $\boldsymbol{y}=\left(y_{i j}\right)_{1 \leq i \leq r, 1 \leq j \leq n_{i}}$ the vector of whole data. The Expectation-Maximization (EM) algorithm is a classical approach to estimate parameters of models with non-observed or incomplete data, Dempster et al. (1977). For non-linear mixed models, the non-observed vector is the individual parameter vector $\boldsymbol{\Theta}=\left(\boldsymbol{\Theta}_{1}, \ldots, \boldsymbol{\Theta}_{r}\right)$ and the complete data of the model is $(\boldsymbol{y}, \boldsymbol{\Theta})$. Let us define the function
$Q\left(\boldsymbol{\psi} \mid \boldsymbol{\psi}^{\prime}\right)=E\left(L_{c}(\boldsymbol{y}, \boldsymbol{\Theta} ; \boldsymbol{\psi}) \mid \boldsymbol{y} ; \boldsymbol{\psi}^{\prime}\right)$, where $L_{c}(\boldsymbol{y}, \boldsymbol{\Theta} ; \boldsymbol{\psi})$ is the log-likelihood of the complete data. At the $m$-th iteration of the EM algorithm, the E step is the evaluation of $Q_{m}(\boldsymbol{\psi})=Q\left(\boldsymbol{\psi} \mid \widehat{\boldsymbol{\psi}}_{m}\right)$, whereas the M step updates $\widehat{\boldsymbol{\psi}}_{m}$ by maximizing $Q_{m}(\boldsymbol{\psi})$. For cases in which the E step has no analytic form, Delyon et al. (1999) introduce a stochastic version of the EM algorithm that evaluates the integral $Q_{m}(\boldsymbol{\psi})$ by a stochastic approximation procedure. The authors prove the convergence of this SAEM algorithm under general conditions if $L_{c}(\boldsymbol{y}, \boldsymbol{\Theta} ; \boldsymbol{\psi})$ belongs to a regular curved exponential family:

$$
L_{c}(\boldsymbol{y}, \boldsymbol{\Theta} ; \boldsymbol{\psi})=-\Lambda(\boldsymbol{\psi})+\langle S(\boldsymbol{y}, \boldsymbol{\Theta}), \Phi(\boldsymbol{\psi})\rangle,
$$

where $\langle.,$.$\rangle is the scalar product and S(\boldsymbol{y}, \boldsymbol{\Theta})$ is the minimal sufficient statistic of the model. The E step is then divided into a simulation step (S step) of the nonobserved data $\boldsymbol{\Theta}^{(m)}$ under the conditional distribution $p\left(\boldsymbol{\Theta} \mid \boldsymbol{y} ; \widehat{\boldsymbol{\psi}}_{m}\right)$ and a stochastic approximation step (SA step)

$$
\begin{aligned}
& \text { of } E\left(S(\boldsymbol{y}, \boldsymbol{\Theta}) \mid \widehat{\boldsymbol{\psi}}_{m}\right): \\
& \qquad s_{m+1}=s_{m}+\gamma_{m}\left(S\left(\boldsymbol{y}, \boldsymbol{\Theta}^{(m)}\right)-s_{m}\right),
\end{aligned}
$$

using $\left(\gamma_{m}\right)_{m \geq 0}$ a sequence of positive numbers decreasing to 0 . The M step is thus the update of the estimate $\widehat{\boldsymbol{\psi}}_{m}$ :

$$
\widehat{\boldsymbol{\psi}}_{m+1}=\arg \max _{\boldsymbol{\psi}}\left(-\Lambda(\boldsymbol{\psi})+\left\langle s_{m+1}, \Phi(\boldsymbol{\psi})\right\rangle\right) \text {. }
$$

For non-linear mixed models, the simulation step is complex because the posterior distribution $p(\boldsymbol{\Theta} \mid \boldsymbol{y} ; \boldsymbol{\psi})$ has no analytical form. In this case, a Monte Carlo Markov Chain is used to simulate $\boldsymbol{\Theta}$. At the $m$-th iteration, the $\mathrm{S}$ step is the simulation of $\boldsymbol{\Theta}^{(m)}$ with use of a MetropolisHastings algorithm which constructs a Markov Chain, with $p\left(\boldsymbol{\Theta} \mid \boldsymbol{y} ; \widehat{\boldsymbol{\psi}}_{m}\right)$ as the unique stationary distribution (see Kuhn and Lavielle (2005) for more details). Kuhn and Lavielle (2005) prove that under general hypotheses, the sequence $\left(\widehat{\boldsymbol{\psi}}_{m}\right)_{m \geq 0}$ obtained by the SAEM-MCMC algorithm converges almost surely towards a (local) maximum of the likelihood. This algorithm is implemented in the Monolix software.

\section{EXPERIMENTAL SETUP}

\subsection{Output variable}

In practice, the tumor volume is estimated from the measurement by an electronic caliper of two distances $d_{1}$ and $d_{2}$ which are supposed to be the small and large axis of an ellipse respectively. The depth axis of the ellipsoid tumor is not accessible to the experimenter. The estimation formula of the tumor volume is then given by

$$
y=\frac{d_{2} d_{1}^{2}}{2} \quad \text { with } d_{1}<d_{2} .
$$

\subsection{Material and method}

Female nude mice were used for tumor implantation. The model of human malignant glioma was obtained using U87 cells, xenografted in mice. Treatments started when tumors reached a volume of $250 \pm 50 \mathrm{~mm}^{3}$ and were based on the combination of topotecan (daily intraperitoneal injection of $0.15 \mu \mathrm{g} / \mathrm{g}, 5$ days/week) with ionising radiation (2 Gy/day, 5 days/week) delivered for four weeks. The 


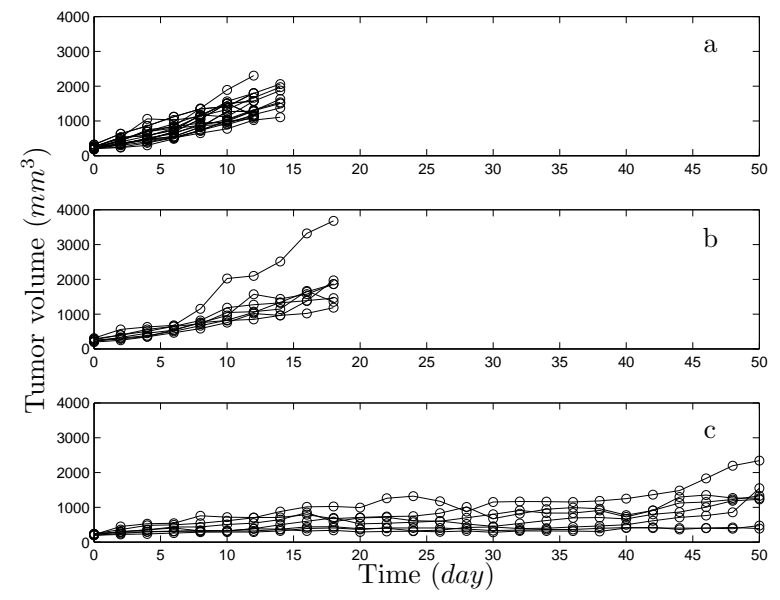

Fig. 2. Longitudinal data sets: tumor volume responses, (a) the control group, (b) radiotherapy group, (c) combined chemotherapy-radiotherapy group

complete biological and medical protocol is defined in Pinel et al. (2006). Measurements were carried out until tumors reached a size of $2 \mathrm{~cm}^{3}$, at which time mice were sacrificed by cervical dislocation.

In this study, the system identification of anticancer responses is decomposed into two successive parts. In a first step, the autonomous growth model $\left(f_{0}\right.$, see $\left.(8)\right)$, composed of two parameters $v_{0}$ and $\alpha$, is identified from the control data set. No covariate is included in this case. Secondly, parameters or more exactly hyper-parameters of the treated tumor model $\left(f_{0}\right.$ and $f_{u}$, see $\left.(10)\right)$ are estimated from the treated data set. The treatment covariate (radiotherapy / combined therapy) is then included. The two identification steps were carried out with Monolix.

\subsection{In vivo data sets}

In vivo kinetic responses of the tumor volume for the control and the two treated groups are presented in Figure 2. The control data set contained 15 kinetic responses while the treated data sets were composed of 7 responses.

\section{IDENTIFICATION RESULTS}

\subsection{Identification of the natural tumor growth}

Identification results of the natural tumor growth, obtained from the control data set, are presented in Table 2 . s.e and r.s.e mean the empirical standard deviation and the empirical variation coefficient respectively. $\omega_{v_{0}}$ and $\omega_{\alpha}$ are two diagonal elements of the covariance matrix $\Omega$, defined in (14). Figure 3 presents a scatterplot of the observed values with the median predicted response and its $90 \%$ prediction interval. The prediction interval is computed with the population parameters in the sample. Some estimated responses using the population parameters and the individual parameters are exhibited in Figure 4 and compared with measured responses. Figure 5 displays the empirical distributions of the population and individual weighted residuals (PWRES and IWRES respectively). Quantilequantile plots are also indicated to assess the gaussian assumption about the output residual (see equation (13)).
Table 2. Estimation results (control group)

\begin{tabular}{|l|c|c|c|}
\hline parameter & estimate & s.e. & r.s.e.(\%) \\
\hline \hline$v_{0}$ & 339 & 19 & 6 \\
\hline$\alpha$ & 0.121 & 0.0038 & 3 \\
\hline \hline$\omega_{v_{0}}$ & 0.193 & 0.038 & 20 \\
\hline$\omega_{\alpha}$ & 0.0575 & 0.039 & 69 \\
\hline \hline$a$ & 130 & 8.5 & 7 \\
\hline
\end{tabular}

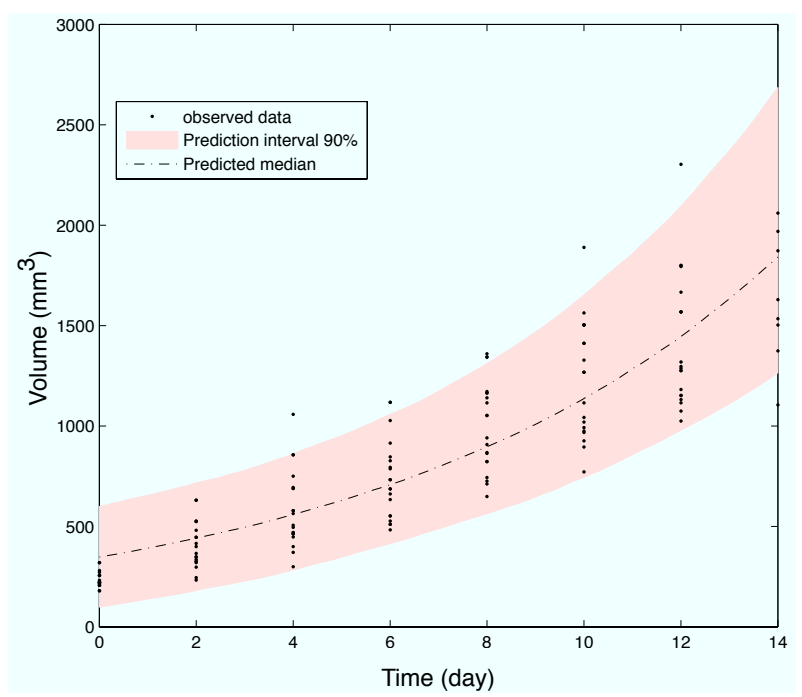

Fig. 3. Predicted response and $90 \%$ uncertainty interval (control group)
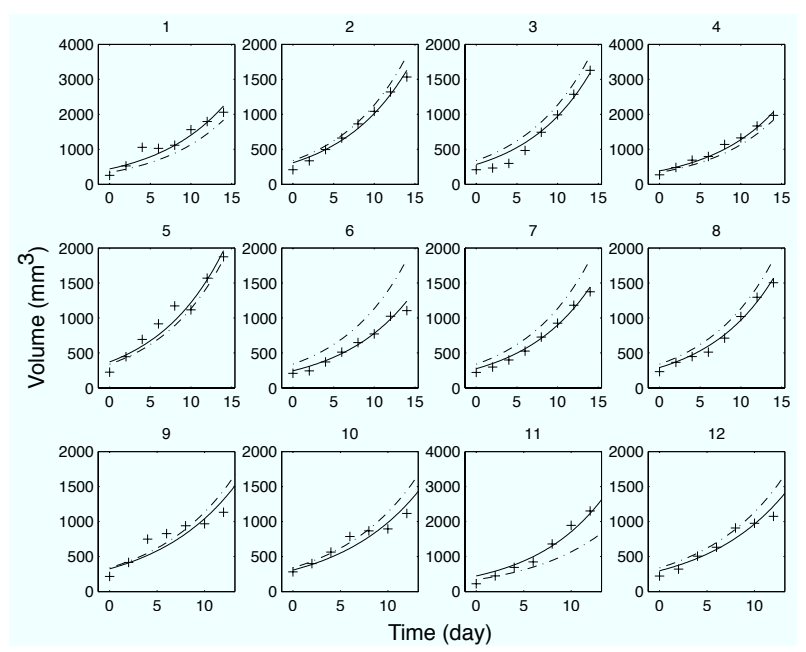

Fig. 4. Measured $(+)$ and estimated responses using the population parameters $(\cdot-\cdot)$ and the individual parameters $(-)$ (control group)

\subsection{Identification of the treated tumor growth}

Identification results of the two treated groups (radiotherapy and combined-therapy) are presented in Table $3 . \beta$ are the coefficients of the treatment covariate. Note in bold text in Table 3 that uncertainty about $u_{0}$ and $\nu$ is too large to consider these estimates as relevant. This result mainly puts into question the choice of the model structure and particularly the dynamical part associated with the treatment. Four estimated responses using the population parameters and the individual parameters are exhibited in Figure 6 and compared with measured responses. It 

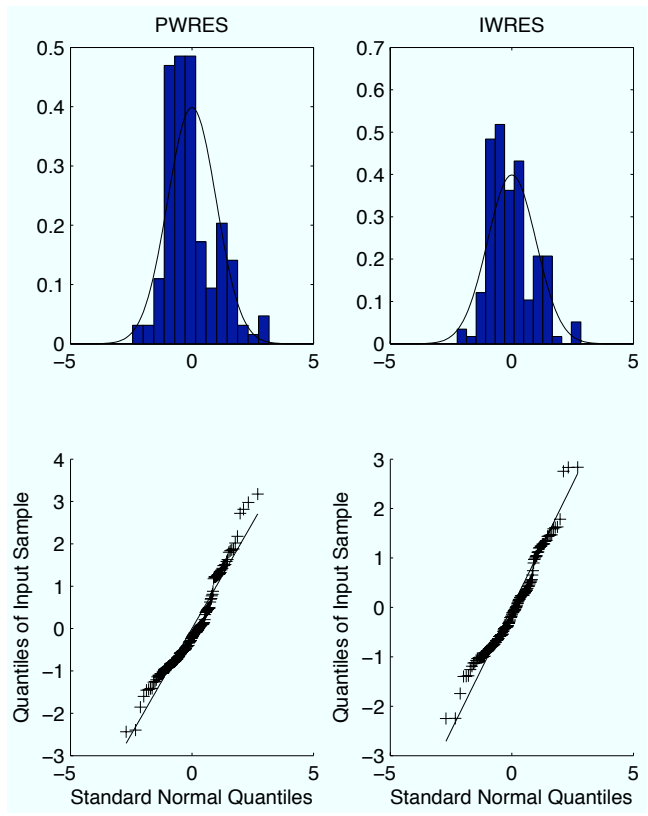

Fig. 5. Empirical distributions of the population and individual weighted residuals and quantile-quantile plots (control group)

is shown that the delayed treatment effect is correctly fitted. Figure 7 displays the empirical distributions of the population and individual weighted residuals and the quantile-quantile plots. As for the control group, there is no significant fact to reject the Gaussian hypothesis about the output residual distribution. Figure 8 displays the empirical distributions of four model parameters $\left(v_{0}, \alpha, u_{0}, \tau\right)$ for the two treated groups.

Table 3. Estimation results (treated group)

\begin{tabular}{|l|r|r|r|}
\hline parameter & est. value & s.e. & r.s.e.(\%) \\
\hline \hline$v_{0}$ & 386 & 34 & 9 \\
\hline$\beta_{v_{0}}$ (CAT_2) & -0.293 & 0.13 & 44 \\
\hline$\alpha$ & 0.0578 & 0.004 & 7 \\
\hline$\beta_{\alpha}$ (CAT_2) & 0.732 & 0.096 & 13 \\
\hline$u_{0}$ & $1.6 \mathrm{e}+04$ & $1.4 \mathrm{e}+05$ & $\mathbf{8 9 3}$ \\
\hline$\beta_{u_{0}}$ (CAT_2) & -159 & $6.4 \mathrm{e}+03$ & $\mathbf{4 . 0 2 e + 0 3}$ \\
\hline$\nu$ & 0.00774 & 0.071 & $\mathbf{9 1 6}$ \\
\hline$\tau$ & 21.4 & 1.3 & 6 \\
\hline$\beta_{\tau}\left(\mathrm{CAT} \_2\right)$ & -0.51 & 0.089 & 17 \\
\hline \hline$\omega_{v_{0}}$ & 0.18 & 0.046 & 25 \\
\hline$\omega_{\alpha}$ & 0.099 & 0.037 & 38 \\
\hline$\omega_{u_{0}}$ & 12.9 & $3.4 \mathrm{e}+07$ & $\mathbf{2 . 6 1 e + 0 8}$ \\
\hline$\omega_{\nu}$ & 0.442 & 4.1 & $\mathbf{9 3 0}$ \\
\hline$\omega_{\tau}$ & 0.129 & 0.036 & 28 \\
\hline \hline$a$ & 118 & 7.8 & 7 \\
\hline
\end{tabular}

\section{DISCUSSION}

Three main points emerge from these estimation results:

- the intracellular damages caused by the anticancer treatment cannot be measured on-line, i.e. without a mouse sacrifice before the end of the experiment. Estimating $u_{0}$ from in vivo data allows to quantify the damage amount. According to Figure 8 and the empirical distribution of $u_{0}$, alterations caused by the combined-therapy are more important than the

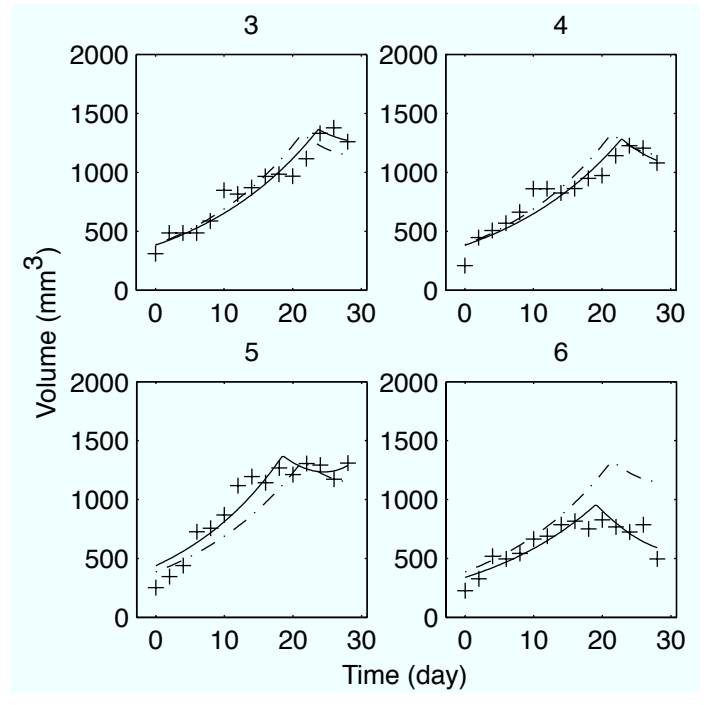

Fig. 6. Measured $(+)$ and estimated responses using the population parameters $(-)$ and the individual parameters $(\cdot-\cdot)$ (treated groups)
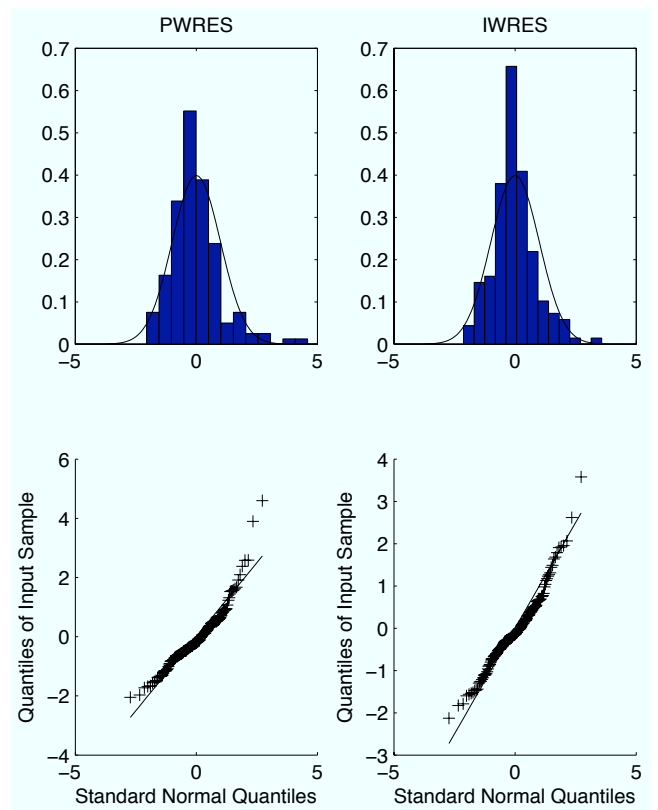

Fig. 7. Empirical distributions of the population and individual weighted residuals and quantile-quantile plots (treated groups)

ones due to the radiotherapy only. However, since the estimated values of $u_{0}$ are very uncertain, this remark should be considered with great care, analysis on more subjects could confirm this fact in the future;

- the anticancer treatment effect on the tumor volume response is well fitted by the proposed model. Two parameters: $\nu$ and $\tau$ of the treatment function $f_{u}$ are meaningful. The first one $(\nu)$ denotes the rate of the volume decrease caused by the treatment. The second one $(\tau)$ informs about the growth delay due to the treatment. Like $u_{0}$, the $\nu$ estimate is very uncertain while $\tau$ is more accurately estimated. The empirical distribution of $\tau$ confirms that the combined therapy increases the treatment time delay compared to the radiotherapy. $\nu$ and $\tau$ describe the most noticeable 

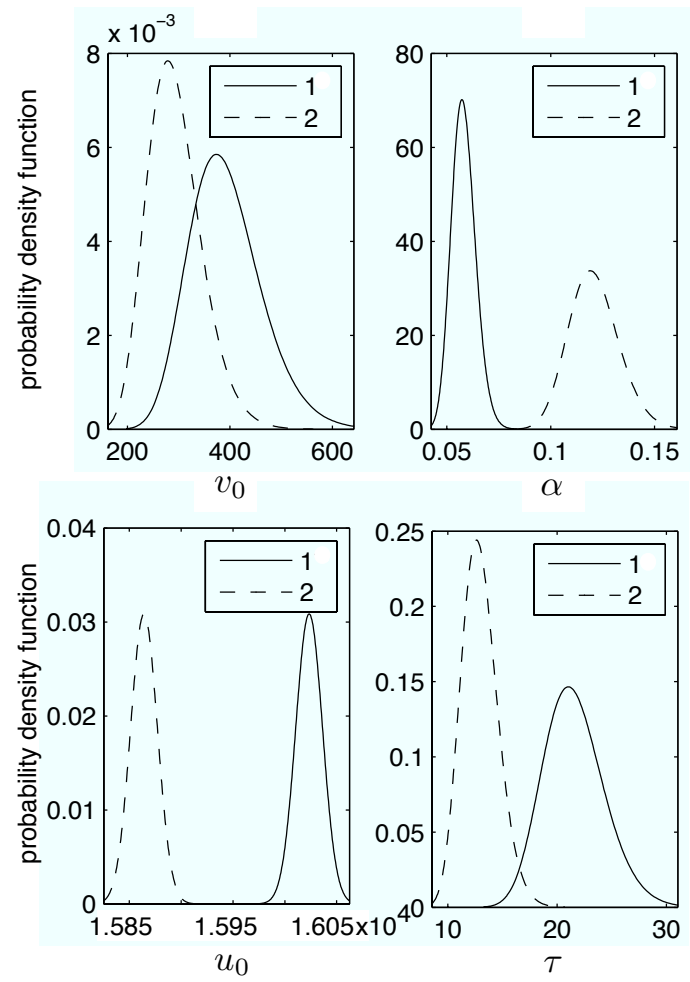

Fig. 8. Estimated distributions of the main model parameters (1:- combined-therapy group, $2:--$ radiotherapy group, )

effect of the therapy on the tumor volume response, but another effect is perceptible in these estimation results;

- indeed, by comparing the empirical distributions of $\alpha$ for the control and treated groups, it clearly appears a significant decrease of its value. The minimum value of $\alpha$ is obtained for the combined-therapy. In other words, this anticancer treatment also slows down the cycle frequency of the cancer cells before to potentially decrease the tumor volume as previously described.

\section{CONCLUSION}

Empirical modeling of tumor growth from in vivo longitudinal data sets was investigated in this paper. The proposed dynamical model structure is composed of two parts associated with autonomous and forced regimes, composed of two and three parameters respectively. Interindividual variability of the tumor growth responses is taken into account by introducing mixed effects into the parametric model. The parameter estimation is carried out by an expectation maximization algorithm based on a stochastic approximation and implemented in Monolix, a free Matlab software. The proposed model showed a good fit of the tumor volume responses. However, the large uncertainty on two parameters put into question the treatment part of the model structure. Nevertheless, the estimated distributions of the other parameters clearly pointed out two specific consequences of the treatments (radiotherapy and combined radiotherapy-chemotherapy) on the tumor growth responses. The first effect is a quasiinstantaneous slowing down of the cancer cell cycle. The second therapeutic action is a delayed decrease of the tumor volume. Perspectives drawn from these results mainly concern model-based control in oncology.

\section{REFERENCES}

J. A. Adam and N. Bellomo. A survey of Models for Tumor-Immune System Dynamics. Modeling and Simulation in Science, Engineering and Technology. Birkhäuser, Boston, 1997.

L. G. de Pillis and A. Radunskaya. A mathematical tumor model with immune resistance and drug therapy: an optimal control approach. Journal of Theoretical Medicine, 3:79-100, 2001.

B. Delyon, M. Lavielle, and E. Moulines. Convergence of a stochastic approximation version of the EM algorithm. Annals of Statistics, 27:94-128, 1999.

A. P. Dempster, N. M. Laird, and D. B. Rubin. Maximum likelihood from incomplete data via the EM algorithm. Journal of the Royal Statistical Society, Series B (Methodological), 39(1):1-38, 1977.

D. Drasdo and St. Höhme. A single-cell-based model of tumor growth in vitro: monolayers and spheroids. Phys. Biol., 2:133-147, 2005.

P. Dua, V. Duab, and E. N. Pistikopoulos. Optimal delivery of chemotherapeutic agents in cancer. Computers and Chemical Engineering, 32:99-107, 2008.

J. J. Fischer. Mathematical simulation of radiation theory of solid tumors. I. Calculations. Acta Radiol. Ther. Phys. Biol., 10:73, 1971.

E. Kuhn and M. Lavielle. Maximum likelihood estimation in nonlinear mixed effects models. Computational Satistics and Data Analysis, 49:1020-1028, 2005.

M. Lindstrom and D. Bates. Nonlinear mixed-effects models for repeated measures data. Biometrics, 46:673687, 1990.

S. Pinel, P. Chastagner, J.-L. Merlin, C. Marchal, A. Taghian, and M. Barberi-Heyob. Topotecan can compensate for protracted radiation treatment time effects in high grade glioma xenografts. J Neurooncol., 76(1): 31-8, 2006.

A. Samson, M. Lavielle, and F. Mentré. Extension of the SAEM algorithm to left-censored data in nonlinear mixed-effects model: application to hiv dynamics model. Computational Statistics and Data Analysis, 51 (3):1562-74, 2006.

A. Samson, M. Lavielle, and F. Mentré. The SAEM algorithm for group comparison tests in longitudinal data analysis based on non-linear mixed-effects model. Statistics in Medicine, 26(27):4860-4875, 2007.

J. Tervo, M. Vauhkonen, and E. Boman. Optimal control model for radiation therapy inverse planning applying the boltzmann transport equation. Linear Algebra and its Applications, 2007.

J. R. Thompson and B. W Brown. Cancer Modeling. Marcel Dekker, 1987.

R. Walter and I. Lamprecht. Thermodynamics and Kinetics of Biological Processes, chapter Modern theories concerning the growth equations, page 143. de Gruyter, I. Lamprecht and A. I. Zotin edition, 1978. 